\title{
Optimization of Pavement Maintenance and Rehabilitation Activities Using Differential Evolution Algorithms
}

\author{
Jia-Ruey Chang
}

\begin{abstract}
A large number of publications have dealt with the computational efficiency of a novel evolutionary algorithm referred to as the differential evolution (DE) algorithm. The DE algorithm is able to optimally adjust the parameters in a dynamic and complex system such as a pavement management system. This study, which is based on real data, identifies maximization of pavement serviceability as the objective to efficiently optimize maintenance and rehabilitation (M\&R) activities using the DE algorithm. It is clear that the DE algorithm is capable of searching based on the objective function under specified constraints for solving the optimization problem regarding pavement $M \& R$ activities.
\end{abstract}

Keywords-optimization, pavement, maintenance and rehabilitation, differential evolution

\section{Introduction}

A pavement provides a steady, fast, and economic surface for road users. Good pavement conditions depend on effective collaboration among planning, design, construction, excavation management, and maintenance and rehabilitation (M\&R) activities. Pavement conditions deteriorate with time owing to internal and external causes such as poor subgrades, defective drainage systems, unstable embankments, problematic quality control, traffic usage, material aging, and varying temperatures. Since pavement defects easily affect road users' safety, pavement engineers have to maintain pavement serviceability above an acceptable level by implementing timely and appropriate $M \& R$ activities [1]. If a pavement defect is not rapidly addressed, it can result in serious problems and significantly shorten the lifespan of the pavement. Hence, the optimization of $M \& R$ activities is very important. However, the resource allocation for $M \& R$ activities is usually performed by a ranking system (a prioritization method) or an optimization model (network optimization) [2] to search for optimal activities for maintaining pavements in a serviceable condition over a period of time. For prioritization methods, some priorityranking criteria or a ranking matrix is used to prioritize $M \& R$ resource allocations to obtain alternative M\&R activities. For network optimizations, a network optimization model with its objective functions and constraints is used to identify the optimal M\&R activities, which is adopted in this study.

Jia-Ruey Chang

National Ilan University

Taiwan
The differential evolution (DE) algorithm is a populationbased direct-search algorithm for global optimization [3]. The DE algorithm was initially proposed for unconstrained, continuous optimization problems. Its basic principle relies on the design of a simple mutation operator based on the linear combination of three different individuals and on a crossover step that mixes the initial and the mutated solutions. The mutation process included in a DE algorithm is not only simple but also important for efficiently obtaining optimal solutions. DE algorithms have been rarely used in pavement engineering and management application. In this study, a network optimization model based on the objective of maximizing pavement serviceability is proposed to identify optimal M\&R activities via the DE algorithm. Real data from the Taiwan Area National Freeway Bureau (TANFB) were collected to conduct an empirical study to reveal and verify the feasibility of the model.

The remainder of this paper is organized as follows. The second section reviews prioritization and optimization in pavement management. The third section briefly reviews the DE algorithm. The fourth section elaborates the model development and conducts an empirical study by using the DE algorithm according to the actual situation in Taiwan. The analytical results are demonstrated and discussed. Finally, the conclusions are summarized in the fifth section.

\section{Review of Optimization in Pavement Management}

Pavement inspections are periodically performed via both man-made and automatic approaches to collect large amounts of pavement condition data. Based on this data, existing pavement conditions are represented using specified indexes such as present serviceability index (PSI), pavement condition index (PCI), and international roughness index (IRI) to assist in $M \& R$ resource allocations. A number of methodologies are commonly adopted to allocate $M \& R$ resources. They are generally categorized into prioritization methods and network optimizations [1, 4-6]:

- Prioritization models are used to categorize and rank the entire pavement sections by using simple priorityranking criteria to establish the ranking of a pavement section without considering the future performance of the pavement. The common ranking factors include pavement type, pavement conditions, traffic volume, pavement age, roughness, friction, and structural capacity. The $M \& R$ resources are allocated according to the ranking and priority assigned to it. The 
Proc. of the Third Intl. Conf. on Advances in Civil and Structural Engineering - CSE 2015

Copyright $\odot$ Institute of Research Engineers and Doctors, USA .All rights reserved.

ISBN: 978-1-63248-057-6 doi: 10.15224/ 978-1-63248-057-6-50

prioritization model is suitable for project-level pavement management.

- $\quad$ Network optimization models identify network M\&R activities. The objectives can be specified as maximizing the performance of a complete pavement network, minimizing the total network cost, and/or minimizing the total $M \& R$ cost subjected to constraints such as acceptable serviceability and budget limits [1]. The pavement condition data are used as inputs to the model while decision variables represent the application of feasible $M \& R$ activities to pavement sections. Further, resource limits act as constraints. A network optimization model is suitable for network-level pavement management that concentrates on the entire pavement network, which uses $M \& R$ performance as objective functions with budget limitations [7].

According to a review of a survey conducted in 1991 [8], the percentage of most U.S. state highway agencies that implement and plan to implement a prioritization model are $77 \%$ and $2 \%$, respectively. This is shown in Table I. Meanwhile, the percentage of states that have and plan to implement a network optimization model are $28 \%$ and $19 \%$, respectively. Note that the total percentage exceeds $100 \%$ as some states implement both a prioritization model and an optimization model. According to Table I, thirteen states use optimization models but just four techniques are used in the optimization models, as shown in Table II. Linear programming is the most common programming technique to allocate M\&R resources. In addition, other programming techniques such as dynamic programming are also proposed [9-12].

TABLE I. METHODOLOGIES FOR M\&R ACTIVITIES USED IN THE U.S.

\begin{tabular}{|l|c|c|}
\hline \multicolumn{1}{|c|}{ Prioritization/Optimization } & Number & Percentage \\
\hline no methodologies adopted & 4 & 0 \\
\hline prioritization model & 36 & 77 \\
\hline plans for prioritization model & 1 & 2 \\
\hline optimization model & 13 & 28 \\
\hline plans for optimization model & 9 & 19 \\
\hline
\end{tabular}

TABLE II. TECHNIQUES USED IN OPTIMIZATION MODELS

\begin{tabular}{|l|c|c|}
\hline \multicolumn{1}{|c|}{ Technique } & Number & Percentage \\
\hline linear programming & 7 & 55 \\
\hline integer programming & 2 & 15 \\
\hline incremental benefit-cost & 2 & 15 \\
\hline marginal cost-effectiveness & 2 & 15 \\
\hline
\end{tabular}

All M\&R activities cannot be funded and implemented within one or even within a few years due to resource constraints. The decision-making task of M\&R activities has to identify specific objectives and constraints. The programming methodology searches for feasible solutions to decision variables that optimally satisfy specific objective functions and constraints. The common objective functions and constraints are summarized in Tables III and IV, respectively. Note that the total percentage in Table III exceeds $100 \%$ as some optimization models have more than one objective function.
TABLE III. OBJECTIVE FUNCTION USED IN OPTIMIZATION MODELS

\begin{tabular}{|l|c|c|}
\hline \multicolumn{1}{|c|}{ Objective Function } & Number & Percentage \\
\hline minimize cost & 8 & 62 \\
\hline maximize area under performance curve & 5 & 39 \\
\hline minimize disutility & 1 & 8 \\
\hline maximize maintenance effectiveness & 1 & 8 \\
\hline
\end{tabular}

TABLE IV. CONSTRAINTS USED IN OPTIMIZATION MODELS

\begin{tabular}{|l|c|c|}
\hline \multicolumn{1}{|c|}{ Constraint } & Number & Percentage \\
\hline budget & 13 & 100 \\
\hline minimal pavement condition requirement & 5 & 39 \\
\hline resources & 2 & 15 \\
\hline other & 5 & 39 \\
\hline
\end{tabular}

This study takes network-level pavement management into account to identify one objective function, namely maximizing pavement serviceability, to determine the optimal M\&R activities via the DE algorithm.

\section{Differential Evolution (DE) Algorithm}

The DE algorithm computes the optimal solution based on the characteristic of a swarm, which strengthens the searching ability in both local and global domains. The DE algorithm has the same mutation, crossover, and selection operations as a genetic algorithm (GA); it also has the same randomlysearching mechanism as a particle swarm optimization (PSO) algorithm. Compared to a GA, the DE algorithm bears the advantages of setting fewer parameters and easy of operations; meanwhile, compared to a PSO, the DE algorithm can obtain more diverse solutions.

The operations of a standard DE algorithm (as shown in Fig. 1) includes five stages, namely initialization, mutation, crossover, selection, and stopping condition verification. Suppose that one would like to minimize a cost objective function $f(X)$, the number of decision variables is $D$. Each of the stages are elaborated in Fig. 1 [13, 14].

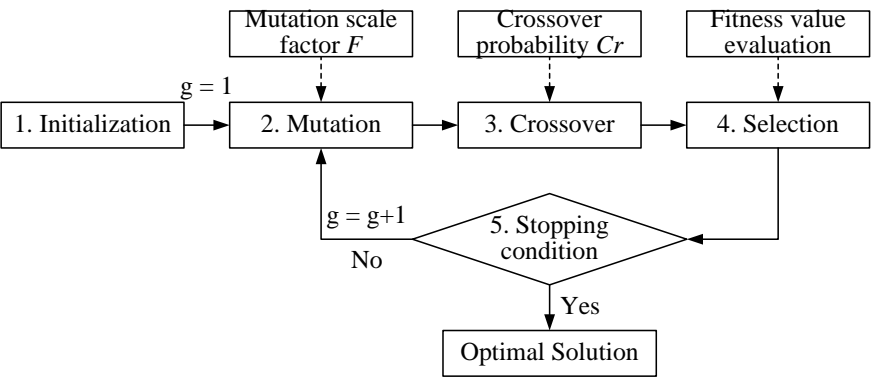

Figure 1. Differential evolution algorithm [14].

- Initialization: the DE algorithm commences the search operation by randomly generating an $N P$ number of $D$-dimensional parameter vectors $X_{i, g}$, where $i=1,2, \ldots, N P$ and $g$ represents the current generation. In the DE algorithm, $N P$ does not change during the optimization process [15]. In addition, the 
initial population, $g=0$, is randomly generated to cover the entire search space.

- Mutation: A vector in the current population is referred to as a target vector. For each target vector, a mutant vector is produced through the following equation [15]:

$V_{i, g+1}=X_{r_{1}, g}+F \cdot\left(X_{r_{2}, g}-X_{r_{3}, g}\right)$

where $r_{1}, r_{2}$, and $r_{3}$ are three random indices and $r_{1}, r_{2}, r_{3} \in[1, N P]$, are integers and are mutually different and $r_{1} \neq r_{2} \neq r_{3} \neq i . \quad F$ is a real number $(F>0)$ and a mutant scale factor that controls the amplification of the differential variation $\left(X_{r_{2}, g}-X_{r_{3}, g}\right)$. Meanwhile, $V_{i, g+1}$ represents the newly created mutant vector.

- Crossover: In order to increase the diversity of the current population, the crossover stage is conducted by exchanging components of the target vector and the mutant vector. In this stage, a new vector named the trial vector, is created. The trial vector is also referred to as the offspring. The following trail vector $U_{j, i, g+1}$ is adopted:

$U_{j, i, g+1}=\left(U_{1, i, g}, U_{2, i, g}, \ldots, U_{D, i, g}\right)^{I}$

$U_{j, i, g+1}=\left\{\begin{array}{l}V_{j, i, g+1}, \quad \text { if } \text { rand }_{j} \leq C r \text { or } j=\operatorname{rnb}(i) \\ X_{j, i, g}, \quad \text { if } \text { rand }_{j}>C r \text { and } j \neq r n b(i)\end{array}\right.$

where $j$ denotes the element index for any vector and rand $_{j} \in[0,1]$ is a uniform random number. $C r$ is a user-defined crossover rate, and $F$ and $C r$ are both generally in the range $[0.5,1.0]$. Meanwhile, $r n b(i)$ is a randomly chosen index from $[1, D]$ which guarantees that at least one parameter from the mutant vector $\left(V_{j, i, g+1}\right)$ is copied to the trial vector $\left(U_{j, i, g+1}\right)$.

- Selection: In order to decide whether the new vector $U$ shall become a population member at generation $g+1$, it is compared to $X_{i, g}$. If vector $U$ yields a smaller objective function value than $X_{i, g}, X_{i, g+1}$ is set to $U$, otherwise the old value $X_{i, g}$ is retained. The selection operator is expressed as follows:

$$
X_{i, g+1}= \begin{cases}U_{i, g} & \text { if } f\left(U_{i, g}\right) \leq f\left(X_{i, g}\right) \\ X_{i, g} & \text { if } f\left(U_{i, g}\right)>f\left(X_{i, g}\right)\end{cases}
$$

- Stopping condition verification: The optimization process terminates when the stopping criterion is met. The stopping criterion can be assigned by a user. In general, maximum generation $\left(G_{\max }\right)$ or maximum number of function evaluations can be adopted as the stopping criterion. When the optimization process terminates, the final optimal solution is obtained.

\section{Iv. Empirical Study: The Optimization of Pavement M\&R Activities via the DE Algorithm}

Since defects on pavements are easily perceived by road users, pavement engineers are responsible for maintaining desired levels of serviceability by implementing appropriate and timely M\&R activities.

\section{A. Analytical Model}

M\&R activities are implemented to improve pavement serviceability. Therefore, based on the actual situation in Taiwan (annual M\&R budget must be completely exhausted every year, that is, any surplus cannot be carried over to the following year), this study identifies one objective, namely maximization of pavement serviceability, for the analytical model. The optimal solution can be obtained via the $\mathrm{DE}$ algorithm by optimizing the objective.

The literature states that surface roughness and surface distress are the two principal factors that are commonly considered for the optimization of pavement M\&R activities. Pavement surface roughness is one of the most important pavement performance measures in pavement construction quality control [16]. Surface roughness may occur as a result of the construction process, road use, or in some cases a combination of both factors [17]. Roughness represents the longitudinal evenness and is indexed by using the International Roughness Index (IRI). The smaller the IRI, the more even the pavement surface is; in other words, the higher the pavement serviceability. This empirical study uses the IRI data collected on a freeway which mainly provides an even pavement for road users. Hence, it is reasonable to use IRI as the factor for optimizing M\&R activities. Note that the objective should present "maximizing pavement serviceability", but since a smaller IRI represents better pavement serviceability, this objective function is minimized. The optimization model and relevant notations are expressed as follows:

Min $\quad f=\sum_{i=1}^{I} \sum_{j=1}^{J}\left(X_{i} \cdot M_{j}\right) \cdot I R I_{i j}$

subject to

$$
\begin{aligned}
& \sum_{i=1}^{I} \sum_{j=1}^{J}\left(X_{i} \cdot M_{j}\right) \cdot C_{j} \cdot A_{i} \leq B \\
& \sum_{i=1}^{I} \sum_{j=1}^{J}\left(X_{i} \cdot M_{j}\right) \leq D \\
& \sum_{j=1}^{J}\left(X_{i} \cdot M_{j}\right) \leq 1 \quad \forall i=1,2, \cdots, I
\end{aligned}
$$

where

$I:$ total number of pavement sections 
Proc. of the Third Intl. Conf. on Advances in Civil and Structural Engineering - CSE 2015

Copyright ( $)$ Institute of Research Engineers and Doctors, USA .All rights reserved.

ISBN: 978-1-63248-057-6 doi: 10.15224/ 978-1-63248-057-6-50

$J$ : total number of M\&R activities (frequently implemented $\mathrm{M} \& \mathrm{R}$ activities in Taiwan are $1.5 \mathrm{~cm}$ milling and overlay, direct overlay, and localized repair, which are denoted as $j=1,2$, and 3 , respectively. Meanwhile, $j=4$ indicates that no $\mathrm{M} \& \mathrm{R}$ activity is required)

$i$ : candidate sections, $i \in\{1,2, \cdots, I\}$

$j$ : applicable M\&R activities, $j \in\{1,2, \cdots, 4\}$

$X_{i} \cdot M_{j}:$ M\&R activity $j$ implemented on section $i$

$C_{j}$ : unit cost of M\&R activity $j$

$I R I_{i j}$ : the roughness of section $i$ implementing M\&R activity $j$

$A_{i}:$ M\&R area of section $i$

$B:$ allowable annual budget

$D:$ the number of $\mathrm{M} \& \mathrm{R}$ projects that a road agency can handle

\section{B. Analytical Data}

The flexible pavements on the third National Freeway in Taiwan are selected as the analytical network, and the analytical data are obtained from the TANFB. The entire length of the network is $67.968 \mathrm{~km}$ (from $042 \mathrm{k}+000$ to $109 \mathrm{k}+698)$. In the network, the length of a 6-lane section is $35.258 \mathrm{~km}$, the length of a 7-lane section is $20.454 \mathrm{~km}$, and the length of an 8-lane section is $11.462 \mathrm{~km}$. The lane- $\mathrm{km}$ and integer mileage are used to divide the network into analytical units. For example, a 1-kilometer section (from $042 k+000$ to $043 \mathrm{k}+000)$ is subdivided into four analytical units since it has four lanes. Based on this principle, the entire network is subdivided into 387 analytical units for the analytical model.

\section{Tools and Parameter Settings for the DE Algorithm}

The EvA2 (an Evolutionary Algorithms framework, revised version 2) [18], as shown in Fig. 2 and Fig. 3, is used to solve the analytical model. EvA2 is a comprehensive heuristic optimization framework with emphasis on evolutionary algorithms implemented in Java. EvA2 integrates several derivation free optimization methods including the DE algorithm.

The relevant parameters using the DE algorithm are listed in Tables V. For each test, the total number of iterations equal to 500 is employed as the stopping criterion when a population of $P=50$ is used. The result is averaged over 10 random runs.
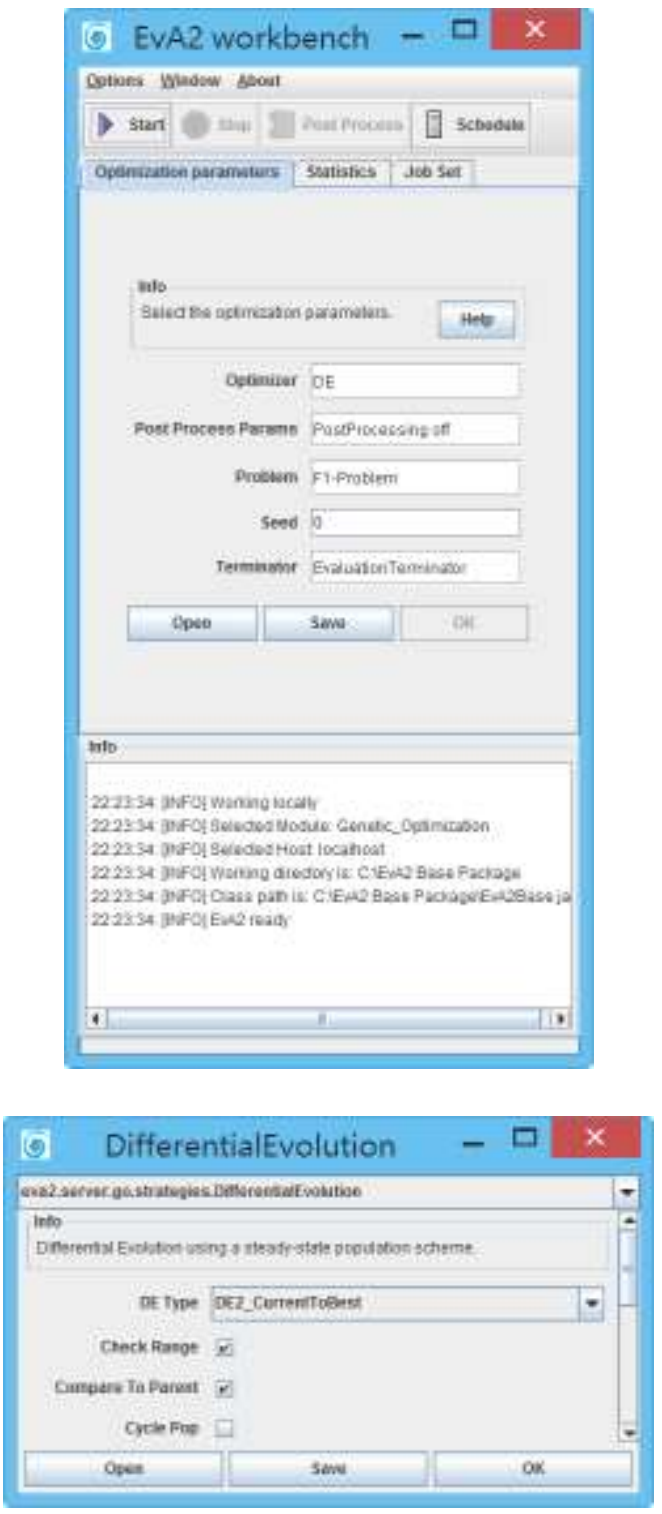

Figure 2. EvA2 base package [18].

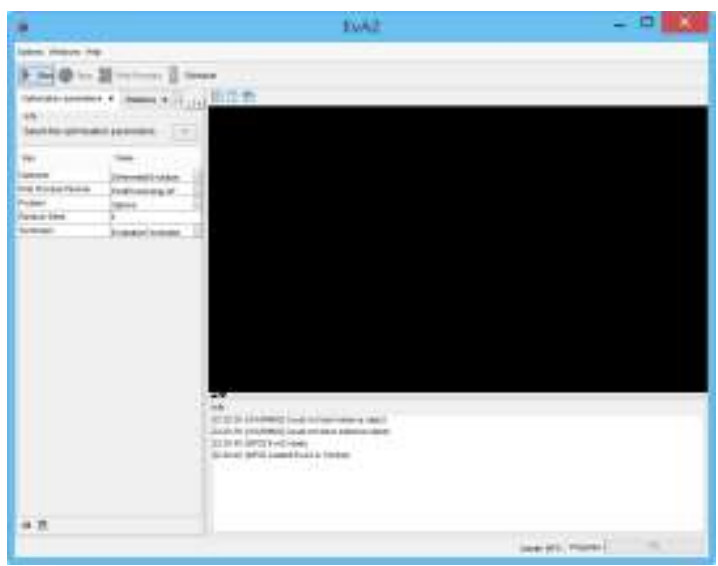

Figure 3. Development build [18]. 
TABLE V. EXPERIMENTAL PARAMETERS USING THE DE ALGORITHM.

\begin{tabular}{|c|l|c|}
\hline Parameter & \multicolumn{1}{|c|}{ Description } & Range \\
\hline$C r$ & crossover rate & 0.9 \\
\hline$F$ & mutant factor & 0.5 \\
\hline$g$ & total number of iterations & 500 \\
\hline$P$ & population size & 50 \\
\hline
\end{tabular}

\section{Results and Discussion}

EvA2 is used to solve the analytical model. The results are obtained while satisfying the objective under the constraints. The maximization of pavement serviceability ( 387 analytical units) is $19,658,235$. It is assumed that the annual budget is US\$ 160,000 (NT\$ 5 million), the road agency can handle twelve projects per year, and every project costs around US\$ 13,000 (NT\$ 420,000).

\section{v. Conclusions}

The DE algorithm searches for the optimal solution based on the characteristics of a swarm. Compared to a GA, the DE algorithm bears the advantages of fewer parameter settings and easy of operations; meanwhile, compared to a PSO, the DE algorithm can yield more diverse solutions. This paper considers the relevant literature and actual $M \& R$ activities in Taiwan, and identifies maximization of pavement serviceability as the objective to efficiently optimize $M \& R$ activities via the $\mathrm{DE}$ algorithm. It is clear that the $\mathrm{DE}$ algorithm is capable of searching based on the objective function under the specified constraints to solve the optimization problem of pavement M\&R activities. Research is currently underway for selecting proper parameters for the DE algorithm in order to yield a more adequate optimal values more efficiently.

\section{Acknowledgment}

The author would like to thank the National Science Council of Taiwan for their financial support provided under the project MOST 103-2221-E-197-009.

\section{References}

[1] Guidelines for Pavement Management System, American Association of State Highway and Transportation Officials (AASHTO), Washington, D.C., 1990.

[2] H. J. Zimmermann, "Fuzzy programming and linear programming with several objective functions," Fuzzy Sets and Systems, vol. 1, pp. 45-55, 1978.

[3] R. Storn and K. Price, "Differential evolution: A simple and efficient adaptive scheme for global optimisation over continuous spaces," Technical Report TR-95-012. International Computer Science Institute, Berkley, 1995.

[4] W. R. Hudson and S. W. Hudson, "Pavement management systems lead the way for infrastructure management systems," Third International Conference on Managing Pavements, Proceedings, vol. 2, pp. 99-112, Transportation Research Board, National Research Council, Washington D.C., 1994.

[5] R. Kulkarni, K. Golabi, F. Finn, E. Alviti, and J. L. Nazareth, Development of a Network Optimization System, vol. I and vol. II, Final Report to Arizona Department of Transportation, 1980.

[6] H. Zimmerman, Pavement Management Methodologies to Select Projects and Recommend Preservation Treatments, National
Cooperative Highway Research Program Synthesis of the Highway Practice 222, Washington, D.C., 1995.

[7] D. T. Phillips, R. L. Laden, and C. V. Shanmugham, Rehabilitation and Maintenance Systems: The Optimization Models, Texas State Department of Highways and Public Transportation, Austin, 1981.

[8] C. I. Federico and T. H. Maze, "Status of pavement management systems and data analysis model at state highway agencies," Transportation Research Record 1397, TRB, National Research Council, Washington, D.C., pp. 1-6, 1993.

[9] A. A. Butt, M. Y. Shahin, S. H. Carpenter, and J. V. Carnahan, "Application of Markov process to pavement management systems at network level," Third International Conference on Managing Pavements, Proceedings, vol. 2, pp. 159-172, Transportation Research Board, National Research Council, Washington D.C., 1994.

[10] K. C. P. Wang and F. Liu, "Fuzzy set-based and performance-oriented pavement network optimization system," ASCE Journal of Infrastructure Systems, vol. 3, no. 4, 1997.

[11] K. J. Feighan, M. Y. Shahin, K. C. Sinha, and T. D. White, “Application of dynamic programming and other mathematical techniques to pavement management Systems," Transportation Research Record 1200, TRB, National Research Council, Washington, D.C., pp. 90-98, 1988.

[12] L. Ningyuan, R. Haas, and H. Michel, "Integer programming of maintenance and rehabilitation treatments for pavement networks," Transportation Research Record 1629, TRB, National Research Council, Washington, D.C., pp. 242-253, 1998.

[13] Y. Liu and F. Sun, "A fast differential evolution algorithm using kNearest Neighbour predictor," Expert Systems with Applications, vol. 38, pp. 4254-4258, 2011.

[14] M. Y. Cheng, N. D. Hoang, and Y. W. Wu, "Hybrid intelligence approach based on LS-SVM and differential evolution for construction cost index estimation: A Taiwan case study," Automation in Construction, vol. 35, pp. 306-313, 2013.

[15] R. Storn and K. Price, "Differential evolution - A simple and efficient heuristic for global optimization over continuous spaces," Journal of Global Optimization, vol. 11, pp. 341-359, 1997.

[16] J. J. Lu, C. Zhu, and J. Pernia, "Performance evaluation of roughness measuring devices to measure ride number and international roughness index," Final Research Report to Florida Department of Transportation, Department of Civil and Environmental Engineering, University of South Florida, 2003.

[17] R. Oliveira de Souza, S. D. Neto, and M. Muniz de Farias, "Statistical analysis between roughness indices and roughness prediction model using neural networks," Improving Pavements with Long-Term Pavement Performance: Products for Today and Tomorrow (Publication No. HRT-06-109), Federal Highway Administration, the American Society of Civil Engineers, 2006.

[18] M. Kronfeld, EvA2 Short Documentation, Last updated May 5, 2011, Retreive from tuebingen.de/software/EvA2/index.html, 2015. http://www.ra.cs.uni-

About Author (s): Jia-Ruey Chang

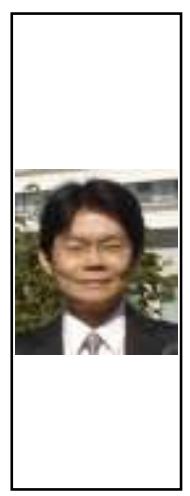

Jia-Ruey Chang received the Ph.D. degree in Civil Engineering from National Central University, Taiwan. He is currently an associate professor at Department of Leisure Industry and Health Promotion in National Ilan University, Taiwan. His research interests focus on the application of advanced methodologies to the pavement engineering and management. Email: 